\title{
Medullary Sponge Kidney and Urinary Calculi Aeromedical Concerns
}

\author{
Jeffrey A. Jones*, Sebastian F. Cherian ${ }^{\#}$, Yael R. Barr ${ }^{\circledR}$, Amber Stocco \\ * NASA/JSC Flight Surgeon, Captain USNR, MAG 41 Medical NAS Ft. Worth \\ MD - Baylor Medical School, Urology Residency - Indiana University \\ Urologic Oncology Fellowship - Indiana University \\ Aerospace Medicine Residency - University of Texas Medical Branch, Galveston \\ Email: jeffery.a.jones@nasa.gov
}

\# Major USAFR

M.D. - Washington University Medical School

Diagnostic Radiology Residency - University of Minnesota

Musculoskeletal Radiology Fellowship - Mayo Clinic, Rochester

Dept of Radiology - Methodist Hospital, Minnesota

Email: sebastian.cherian@parknicollet.com

${ }^{\circledR}$ M.D. - Sackler School of Medicine, Tel-Aviv University

MPH candidate - University of Texas Medical Branch, Galveston

Internal Medicine/Aerospace Medicine Residency (currently) - University of Texas Medical Branch

Email: yrbarr@utmb.edu

\footnotetext{
MD - University of Oklahoma Medical School

Pediatrics Residency - Baylor College of Medicine
}

Pediatric Neurology \& Developmental Neuroscience Fellowship (currently) - Baylor College of Medicine

Email: astocco@bcm.tmc.edu 


\section{Correspondence to:}

Jeffrey A. Jones, MD

NASA/JSC SD2

2101 Nasa Rd\#1

Houston, TX 77058

Phone: 281 483-4418, Fax: 281 483-2224

\section{Manuscript metrics:}

Word count for Abstract - 129

Word count for Narrative Text - 2368

Number of References - 29

Number of Tables - 0

Number of Figures - 2

Keywords:

Sponge kidney, Aerospace medicine, Weightlessness, Nephrolithiasis

\section{Suggested short title:}

MSK Calculi in Aviators 


\begin{abstract}
:
Medullary Sponge Kidney (MSK) is a benign disorder associated with renal stones in $60 \%$ of patients. Patients frequently have episodic painless hematuria but are otherwise asymptomatic unless renal calculi or infections complicate the disease. Nephrolithiasis is a relative, but frequently enforced, contraindication to space or other high performance flight. Two case reports of asymptomatic NASA flight crew with MSK and three cases of military aviators diagnosed with MSK are reviewed, all cases resulted in waiver and return to flight status after treatment and a vigorous follow up and prophylaxis protocol. MSK in aviation and space flight necessitates a highly case-by-case dependent evaluation and treatment process to rule out other potential confounding factors that might also contribute to stone formation and in order to re-qualify the aviator for flight duties.
\end{abstract}




\section{Introduction:}

The United States military and NASA maintain medical standards for aviation. The purpose of these standards is to identify diseases that pose a significant risk in the often harsh and uncompromising aerospace environments. The medical standards are not static; and are subject to change as aeromedical research allows more informative risk management. We review two case reports of NASA aviators with medullary sponge kidney. We will discuss this condition, specifically exploring its predilection to predispose these individuals to renal stones. Also addressed are the subsequent aeromedical disposition and suggestions as to what screening, testing, and precautions should be taken in the future.

Medullary sponge kidney (MSK) is a benign cystic disorder characterized by diffuse cystic dilatation of the collecting ducts limited to the renal medulla. Lenarduzzi first described dilatation of the renal pyramids by excretory urography in 1939 (15). A decade later Cachii and Ricci classified and described MSK as a disease, resulting in MSK's eponym Cachii-Ricci Disease or its other synonym Tubular Ectasia (2). The incidence of MSK varies from 1 in 5000 to 1 in 20,000 in the general population $(10,27)$ and from 1 in 200 to 1 in 20,000 of all excretory urograms $(18,23,27)$. This condition is generally discovered in patients in their third to sixth decade (6). It is more common in men and Caucasians but has a higher rate of complications from calculi in women $(1,5,27)$. The pathogenesis of MSK remains unknown but most authors agree it represents a developmental defect of the collecting tubules $(6,27)$. Diagnosis of this condition is most commonly made by intravenous pyelogram (IVP) which shows collections of contrast adjacent to calices with varying degrees of enhanced pyramidal blush (27) (a.k.a. "paintbrush," "fan-shaped," "bunches of flowers," "cluster of grapes," "mosaic pattern" or "puddling"). Ekstrom reviewed 44 cases of MSK and found the urogram to be 
"roentgenologically pathonomonic" in all but one (5). Pyrah has classified this disease into three groups: 1) patients who were asymptomatic but had diagnostic findings by IVP, 2) patients with nephrocalcinosis and symptoms and 3) patients with frank renal calculi and symptoms (25). The disease is generally pan renal and bilateral (75\% bilateral). Case reports of segmental MSK are uncommon in the literature $(1,5,7,9,19,23)$.

MSK is usually asymptomatic and discovered when an infection or stones complicate the condition. As a rule MSK does not grossly impair renal function $(6,25,27)$. The most common chief complaints include hematuria (10\%-55\%), renal colic (11\%-60\%), lumbar pain (33\%), pyuria (18\%), chronic pyelonephritis $(5 \%-16 \%)$ and urinary symptoms $(11 \%-33 \%)(1,5,6)$. Approximately two thirds of patients with MSK also have impaired medullary concentrating ability with polyuria $(6,27)$. Type I absorptive hypercalciuria has been a well documented but poorly understood association with MSK (7,27). Much more rarely, Renal Tubular Acidosis Type II has been associated with MSK (27).

Renal stone incidence in MSK is controversial as non-stone forming individuals are less likely to undergo excretory urograms. The reported range of stone formation has varied from $40 \%-94 \%$ of those incidentally radiographically diagnosed with MSK $(1,5,16,17)$. Lifetime morbidity of $60 \%$ is the most commonly quoted number, with $50 \%$ being symptomatic within five years of diagnosis. These calculi most commonly are due to a combination of urinary stasis within the dilated collecting tubules (1) and additional metabolic or structural abnormalities (8). Ten percent of those with symptomatic MSK have a poor renal function prognosis due to intractable stones and UTIs (27). 


\section{Aeromedical Case Histories:}

The first case is a male NASA flight crew member who presented with asymptomatic microscopic hematuria on routine annual flight physical examination. IVP was performed, which showed no evidence of calculi, but was consistent with classic findings for the renal tubule ectasia of MSK (Figure 1). A renal stone risk profile (RSRP) was obtained. A total of 2.65 liters of urine over a 24 hour period revealed 1,778 $\mathrm{mg}$ of creatinine (indicative of a full 24 hour collection), calcium $120 \mathrm{mg}$, oxalate $30.1 \mathrm{mg}$, uric acid $530 \mathrm{mg}$, pH 5.6, sodium $109 \mathrm{mEq}$, sulfate $20.4 \mathrm{mmol}$, phosphate $1092 \mathrm{mg}$. All parameters were within normal limits, however the $\mathrm{pH}$ and phosphate limits were at the upper limit of normal. The relative supersaturation for calcium oxalate, brushite, sodium urate, uric acid and magnesium-ammonia-phosphate were all within normal range. Citrate and magnesium (600 $\mathrm{mg}$ and $87 \mathrm{mg}$ respectively) were both in normal inhibitory concentrations. The overall conclusions from RSRP deemed the patient to be at no increased metabolic risk for stone formation.

Due to the absence of findings of metabolic stone risk factors, the patient received a waiver for short duration space flight assignments, but due to the natural history of MSK the patient was not waived for long duration flight assignments (defined as greater than 30 days), such as those on the International Space Station. Annual follow up with RSRP and imaging was also required. The patient has been asymptomatic and without calculi to the present.

In the second case a NASA pilot presented with episodic painless hematuria and pyuria with $1+$ calcium oxalate crystals on urinalysis. Nephrotomograms with pyelogram revealed an $8 \mathrm{~mm}$ calcification in the upper pole of the left kidney and a $3 \mathrm{~mm}$ calculus in the lower pole of the right kidney. The patient was disqualified from flight. 
The patient underwent two courses of bilateral treatment with extracorporeal shock wave lithotripsy. Post treatment renal tomography revealed residual calcification in the left upper pole, which was felt to be within the parenchyma of the kidney in the ectatic tubules of a segmental form of medullary sponge kidney. The remaining kidney had normal tubular structure. These conclusions were validated by a left retrograde pyelography concomitant with a fine cut, non-contrast CT scan.

A metabolic stone evaluation revealed normal serum electrolytes, calcium, and uric acid levels. Two of three 24-hour urine collections revealed mildly elevated urine calcium at $366 \mathrm{mg}$ (normal $<250 \mathrm{mg}$ ) and one increased uric acid level giving a slightly increased risk on RSRP. The aviator was felt to have an incomplete variant of Type I absorptive hypercalcuria producing the minimal elevation in urinary calcium without supersaturation.

A NASA Aeromedical Board voted, based on the removal of calcification from the collecting system, to return the patient to full flight status (as primary pilot) in dual piloted aircraft. There was no restriction on calcium intake but aggressive hydration was deemed mandatory, as well as close follow-up of urinalysis, serum chemistries, 24 hour urine collections, a plain abdominal radiograph (KUB) every 3 months, and a renal ultrasound or excretory urogram with tomograms every 6 months. Any progression of calcification, worsening pyuria/hematuria or increase in RSRP or calcium oxalate saturation index would have called for re-evaluation with possible disqualification from primary pilot status, or additional required intervention (e.g. thiazide diuretics, dietary restrictions, or inhibitor supplements).

Follow-up interval evaluations have shown no genitourinary symptoms, no pyuria, no worsening of microhematuria, and no progression of the calcifications on imaging. His serum creatinine is normal at $0.7 \mathrm{mg} / \mathrm{dL}$, as well as a serum uric acid of $5.5 \mathrm{mg} / \mathrm{dL}$ and calcium of 9.2 
$\mathrm{mg} / \mathrm{dL}$. The patient is currently on a low animal protein diet, has increased his oral hydration, and avoids any periods of prolonged heat exposure on take off or landing that might predispose to dehydration.

Three additional cases $(7,9,19)$ were found in the literature of USAF aviators, all with a segmental form of MSK. Two of the three received a segmental nephrectomy. The third had a radical nephrectomy as IVP, US, and CT all indicated a space-occupying lesion without calcification. This lesion on pathologic examination revealed unilateral matrix material in ectatic tubules consistent with segmental MSK. Unlike the NASA case reports, which were asymptomatic, all of these patients presented with flank pain, one had a past medical history significant for pyelonephritis and the other had multiple UTIs and stones. Post surgery all of these individuals were able to return to full flight status within one year. 


\section{Discussion:}

The extreme environments of high performance aviation and space travel, combined with the grave consequences of medical emergencies in these environments, requires the DOD, FAA, and NASA to have strict medical standards for pilots. The decision to waiver disqualifying medical conditions is a balance between the potential risks of a medical emergency with the loss of service of a highly trained pilot. If MSK were suspected on initial screening of an astronaut candidate, and MSK was confirmed on IVP, it would be disqualifying. The development of urinary calculi in DOD or NASA pilots, who have been previously screened for stone disease prior to selection, is felt to be in most cases environmentally-induced. Screened individuals are not at the same risk level as the general population due to the vigorous selection process. However, due to the sporadic nature and variable presentation of MSK, these patients were not detected on screening ultrasound. Therefore, their occupational environment would only partially contribute to the development of their calculi.

In the space environment, this risk of calculi formation is increased both in- and postflight by multiple metabolic factors $(28,29)$. Osteoclastic activity is dramatically increased and grossly results in a one to one and a half percent loss of bone mass per month $(11,13)$. In comparison, a severely osteoporotic postmenopausal woman loses that same amount in one year $(11,13)$. This results in the hypercalciuria of microgravity. Additionally, hypocitraturia, low urine $\mathrm{pH}$, a $10 \%-15 \%$ decrease in plasma volume and lower urine volume $(12,28,29)$ resulting from the microgravity environment also predispose to stone formation. This has resulted in one in-flight urinary calculus and more then ten post-flight calculi in the combined Russian and US space flight experience. 
On the other hand, the USAF and US Navy (USN) have extensive experience with renal calculi in aviators. In the USAF the history of a single uncomplicated renal calculus is not medically disqualifying for flight, and persons with a history of multiple stones may even be returned to flying duty (4). USN aviators with a history of an uncomplicated renal stone must wait 12 weeks before returning to flying status, after suitable therapy (20). These approaches seem to be justified as a review of naval aviators revealed a reoccurrence rate of only $2 \%$ after a single stone (26) verses $33 \%+$ expected in the general population and $75 \%$ reoccurrence if untreated.

However, often these pilots are removed from USAF Class I, or USN Service Group 1 or 2 statuses, and restricted to multipiloted, non-high performance aircraft, where the risk from acute renal/ureteral colic would be much less devastating. The standards affecting aviator and crew qualification status $(4,20)$ do not allow renal anomalies or diseases that produce significant renal dysfunction or urinary calculi, so these conditions require waiver on a case-by-case basis.

Because of the relative rarity of MSK, experience with this condition within the aerospace medicine community is limited. However, there is a growing body of evidence that indicates the majority of aviators with MSK can continue their flying duties with acceptable risk. From 1987 to 2000, nine naval and marine aviators were found to have MSK (20). Six received waivers (five presented with renal stones and one with recurrent hematuria); none of the six have had subsequent complications due to MSK. Three aviators were denied waivers due to retained or recurrent stones. From 1970 to 2000 the USAF has granted waivers to 29 aviators with MSK. Two were later permanently disqualified for recurrent calculi (4).

Hsu et al's research suggests that "physiologic environment of the urine" resulting in urinary stasis is alone unlikely to cause stone formation but usually must be superimposed on 
other metabolic or physiologic abnormalities (8). Some of these metabolic abnormalities are induced in a microgravity environment, and the question still remains if these microgravity changes are sufficient to cause stone formation in those with MSK, or if a third factor is needed.

An association between MSK and hypercalciuria has been reported as far back as 1959 by Ekstrom (5). O'Neill in his series of 17 cases found that 59\% of patients with MSK also had absorptive hypercalciuria (21). The abnormality in absorptive hypercalciuria is excess small intestinal calcium absorption, which leads to an increase in urine calcium $(22,24)$. The exact pathogenic mechanism for the enhanced absorption remains unknown. In the majority of patients with this disorder, absorption of calcium is independent of vitamin D concentration (14). This disorder can be treated with sodium cellulose phosphate (24).

In conclusion, we have multiple NASA and DOD aviators diagnosed with MSK, a condition well known to increase urinary stone and infection risk. Space flight has additional microgravity risk factors pre-disposing to stones. Evaluation of these individuals on a case-bycase level should weigh the extent of the MSK (i.e. segmental vs. pan renal/bilateral disease), metabolic abnormalities (e.g. hypercalciuria), structural abnormalities (e.g. diverticuli), and past symptoms (prior stones, infections, extensive hematuria, etc). The majority of patients with MSK will develop stones or infection, but urinary stasis in and of itself may not be sufficient to be the only etiology. Depending on secondary predisposing factors there may be a need to intervene by: altering diet (e.g. decreased oxalate or uric acid containing foods such as red meat, decreased sodium intake), increasing hydration ( $>2$ liters per day minimum), avoiding excessive mineral supplements, decreasing caffeine intake, and pharmaceutical therapy (e.g. thiazide diuretics, sodium cellulose phosphate). MSK aviators, if waived for flight duty, must have close follow up with frequent RSRP analysis, imaging, and monitoring for other signs and symptoms 
of pathology. If stones occur, the patient should be restricted from flight until treatment renders the collecting system free of stones, realizing that calcification may persist in the parenchyma, which is not necessarily disqualifying. Partial nephrectomy to remove the severely affected regions of segmental MSK (3) have as presented above resulted in return to flight status in less than twelve months. Surgical treatment is not indicated for the mere presence of stones in the general population (3). In the aerospace community, however, if prompt return to flight status is deemed necessary than surgery may be the most expeditious means of qualifying the aviator for waiver. The risk of partial nephrectomy in the case of segmental MSK must be carefully considered on a case-by-case basis.

\section{Legend to figures:}

Figure 1. IVP showing characteristic bilateral dilated tubules of MSK with contrast blush extending from calices.

Figure 2. CT depicting calculi of MSK in the right kidney parenchyma. 


\section{References:}

1. Abeshouse BS, Abeshouse GA. Sponge kidney: a review of the literature and a report of five cases. J Urol 1960; 84: 252-67.

2. Cacchi R, Ricci V. Sur une rare maladie kystique multiple des pyramides renales le "Rein en eponge". J d’Urol 1949; 55: 499-519.

3. Cohen TD, Preminger GM. Management of calyceal calculi. Urol Clin North Am 1997; 24(1):81-96.

4. Department of the Air Force. Aeromedical waiver guide (formerly AFPAM 48-132), 2003 (updated 21 Oct 2003). Washington, DC: U.S. Air Force; 2003.

5. Ekstrom T, Engfeldt B, Langergren C, Lindvall N. Medullary sponge kidney. Stockholm: Almqvist and Wiksell, 1959.

6. Gillennwater JY, Grayhack JT, Howards SS, Mitchell ME, eds. Adult and Pediatric Urology. $4^{\text {th }}$ edition, Philadelphia: Lippincott Williams \& Wilkins; 2002:863-65.

7. Higashihara E, Nutahara K, Tago K, Ueno A, Niijima T. Unilateral and segmental medullary sponge kidney: renal function and calcium excretion. J Urol 1984; 132(4):7435.

8. Hsu TH, Streem SB. Metabolic abnormalities in patients with caliceal diverticular calculi. J Urol 1998; 160(5):1640-2.

9. Kaver I, Flanders EL, Kay S, Koontz WW Jr. Segmental medullary sponge kidney mimicking a renal mass. J Urol 1989; 141(5):1181-3.

10. Kuiper JJ. Medullary sponge kidney. In: Gardner KD Jr, Ed. Cystic Diseases of the Kidney. New York: Wiley; 1976. 
11. Lang T, LeBlanc A, Evans H, Lu Y, Genant H, Yu A. Cortical and trabecular bone mineral loss from the spine and hip in long-duration spaceflight. J Bone Miner Res 2004; 19(6):1006-12.

12. Leach CS, Rambaut PC. Biochemical observations of long duration manned orbital spaceflight. J Amer Med Women’s Assoc 1975; 30(4):153-72.

13. LeBlanc A, Schneider V, Shackelford L, West S, Oganov V, Bakulin A, et al. Bone mineral and lean tissue loss after long duration space flight. J Musculoskelet Neuronal Interact 2000; 1(2):157-60.

14. Lee PH, Menon M. Etiology of Renal Stones. AUA Update Series 1988; 7: lesson 5.

15. Lenarduzzi G. Reporto pielografico poco commune (dilatazione delle vie urinarie intrarenali). XIV Riunione dei Radiologi Veneti, Rovigo, 1938. Radiol Med (Torino) 1939; 26: 346-7.

16. Lindvall N. Roentgenologic diagnosis of medullary sponge kidney. Acta Radiol 1959; 51(3):193-206.

17. Lowen W, Smythe AD. Cystic disease of the renal pyramids (Medullary Sponge Kidney). Clin Radiol 1964; 15:271-8.

18. Mayall GF. The incidence of medullary sponge kidney. Clin Radiol 1970; 21(2):171-4.

19. Modarelli RO, Wettlaufer JN. Surgically documented segmental medullary sponge kidney: case report. J Urol 1977; 117(2): 244-5.

20. Naval Aerospace Medical Institute. U.S. Navy Aeromedical Reference and Waiver Guide 2004. http://www.nomi.med.navy.mil/NAMI/WaiverGuideTopics

21. O’Neill M, Breslau NA, Pak CY. Metabolic evaluation of nephrolithiasis in patients with medullary sponge kidney. JAMA 1981; 245(12): 1233-6. 
22. Pak CY. Physiological basis for absorptive and renal hypercalciurias. Am J Physiol 1979; 237(6):F415-23.

23. Palubinskas AJ. Medullary sponge kidney. Radiology 1961; 76:911-9.

24. Preminger GM. Medical management of urinary calculus disease Part II: Classification of metabolic disorders and selective medical management. AUA Update Series 1995; 14:1esson 6.

25. Pyrah LN. Medullary sponge kidney. J Urol 1966; 95(3): 274-83.

26. Rocercio PV, Ferrara W. Urolithiasis and naval aeromedical disposition. Aerospace Medical Association Annual Scientific Meeting. Seattle, Washington, 1998.

27. Walsh PC, Retik AB, Vaughan ED, Wein AJ, Kavoussi LR, eds. Campbell's Urology. $8^{\text {th }}$ ed. Philadelphia: Saunders; 2002: 1974-6.

28. Whitson PA, Pietrzyk RA, Pak CYC. Renal stone risk assessment during Space Shuttle flights. J Urol 1997; 158(6): 2305-10.

29. Whitson PA, Pietrzyk RA, Pak CY, Cintron NM. Alterations in renal stone risk factors after space flight. J Urol 1993; 150(3): 803-7. 